\title{
EFECTO DE LA DENSIDAD POBLACIONAL DE CAMINADORA (Rottboellia cochinchinensis L. SW. Clayton) SOBRE EL CULTIVO DE MAÍZ ICA V- 109
}

\author{
EFFECT OF THE POPULATION DENSITY OF ITCHGRASS \\ (Rottboellia cochinchinensis L. SW. Clayton) ON THE \\ CULTIVATION OF MAIZE ICA V - 109
}

\author{
Jaime A. Rivera ${ }^{1}$, Roberto E. Cabrales ${ }^{1}$ y Rafael A. Montoya ${ }^{1}$ \\ Recibido para evaluación: Julio 15 de 2007 - Aceptado para publicación: Noviembre 1 de 2007
}

\begin{abstract}
RESUMEN
Con el objetivo de evaluar el comportamiento fisiológico de la caminadora (Rottboelia Cochinchinensis (L) Sw Clayton), en diferentes poblaciones y su efecto en competencia sobre la variedad de maíz ICA-V-109 en la zona del Sinu medio, se instaló el ensayo en predios de la Universidad de Córdoba, Municipio de Montería, cuyas coordenadas son $8^{\circ} 48^{\prime}$ de Latitud Norte y $75^{\circ} 52^{\prime}$ de Longitud Oeste con respecto al Meridiano de Greenwich con una altura de 14 . m.s.n.m con una $\mathrm{HR}$ del $80 \%$, temperatura promedio de $28{ }^{\circ} \mathrm{C}$ y precipitación promedio anual de $1.200 \mathrm{~mm}$, el experimento constó de cuatro tratamientos $\left(0,2,4,8\right.$ malezas $\left.\mathrm{m}^{-2}\right)$, sembrados en competencia con el cultivo, bajo un diseño completamente al azar. Las variables evaluadas fueron: Altura de planta, número de hoja, numero de nudos, longitud de nudos, área foliar, masa seca, y grosor del tallo, índices fisicotécnicos y componentes de rendimiento para el cultivo. Los resultados sugieren que la interferencia de la caminadora provocó pérdidas significativas en rendimientos de maíz de $514 \mathrm{Kg} \mathrm{ha}^{-1}$ entre el testigo y el tratamiento de 4 malezas por $\mathrm{m}^{2}$. Además la planta de caminadora presentó su alta expresión fenológica teniendo en cuenta los índices fisicotécnicos como lo es la TAN, TAC, RAF, DAF, IAF a los 54 y 56 días coincidiendo con el inicio de floración y llenado del grano del cultivo de maíz, disminuyendo las condiciones de productividad y eficiencia fotosintética en la acumulación de foto asimilados.
\end{abstract}

Palabras clave: Reducción del rendimiento, competencia, caminadora, crecimiento.

\begin{abstract}
The effect of itchgrass (Rottboellia cochinchinensis (L.) W.D. Clayton), at different populations on maize crop variety ICA-V-109 in the Mid Sinu Valley was evaluated. Four treatments (0, 2, 4, 8 itchgrass plant $\left.\mathrm{m}^{-2}\right)$ in competition with the crop, were established using a complete randomized design. The evaluated variables were: plant height, number of leaves, number of knots, node's length, foliar area, dry weight, and stem thickness, physiological indexes and yield components were also evaluated. The results suggest that itchgrass
\end{abstract}

${ }^{1}$ Universidad de Córdoba, Departamento de Ingeniería Agronómica y Desarrollo Rural, Carrera 6 No. 76 - 103, Montería, Colombia, Telefax (4) 786 0255, Email: rmontoya@sinu.unicordoba.edu.co 
competition caused significant yield compared with the control treatment. The itchgrass plant also showed a high physiologic expression, respect to physiological indexes such as NAR (net assimilation rate), AGR (absolute growth rate), FAR (foliar area relation), LAF (foliar area duration), FAI (foliar area index) at 54 and 56 days coinciding with flowering and grain filling of the corn ear, reducing productivity and photosynthesis efficiency for carbohydrate accumulation.

Key words: Yield reduction, competition, itchgrass, growth.

\section{INTRODUCCIÓN}

La concepción generalizada es que las arvenses compiten de forma directa con los cultivos por luz, agua, nutrientes, espacio y de forma indirecta causan pérdidas económicas al afectar los costos de producción y disminuir la calidad de los productos (Arrieta, 1996). En Colombia, las malezas causan reducción de los rendimientos de maíz entre $10 \%$ y $84 \%$, lo cual está directamente relacionado con el tipo, clase y número de arvenses presentes en el cultivo. Con miras a disminuir los altos porcentaje de reducción en la producción que ocasionan las arvenses, es prioridad determinar la época en la cual estas compiten relevantemente con el cultivo (Rivero, 1996).

Según Klingman y Ashton (1989), la capacidad de competencia que tienen las malezas se debe en gran parte a la facilidad con que ellas se adaptan a diversos medios y condiciones, ya que poseen ciertas características tales como: ciclo de vida parecido al del cultivo, rápido desarrollo, plasticidad de sus poblaciones, germinación dispareja, producción de inhibidores, cantidad de semilla y fácil adaptación a las variaciones del medio ambiente. La competencia por $\mathrm{CO}_{2}$ aún se encuentra en discusión, y parece ser de poca importancia a nivel práctico, sin embargo se ha encontrado que la mayoría de las malezas tienen un índice se saturación de $\mathrm{CO}_{2}$ mas bajo que el de algunas plantas cultivadas, lo cual les permite ser mas eficientes en le proceso de fotosíntesis (CIAT, 1990). En estudios realizados por López y Rivero (1996), a nivel de monocultivo la $R$. cochinchinensis, presenta su máxima Tasa Absoluta de Crecimiento a los 53 días después de emergida existiendo una relación directa con el área foliar en ese mismo estado de desarrollo; en cuanto a la Tasa de Crecimiento Relativo su mayor valor se presentó en el intervalo de 0 a 15 días después de emergida, además la TAN como indicador de los fotoasimilados promedios, mostró que la maleza presenta su mayor eficiencia fotosintética promedia hasta los 45 días después de la emergencia (d.d.e.). Partiendo de ese punto, es básico determinar el grado de interferencia de la caminadora en diferentes densidades de población sobre el cultivo de maíz ICA-V-109 en la zona de Montería, Córdoba, con base en el comportamiento fisiológico de la maleza en competencia con el maíz apoyado de un ANACRES, adicionándole la evaluación de los efectos de la densidad de la caminadora sobre los rendimientos del maíz y determinar la capacidad competitiva del maíz con la maleza, basado en rendimiento; con miras a establecer un manejo técnico programado y protección del medio ambiente, logrando una agricultura competitiva, viable y sostenible.

\section{MATERIALES Y MÉTODOS}

La presente investigación de tipo experimental, se realizó en las instalaciones de la Universidad de Córdoba, ubicada bajo las siguientes condiciones: Latitud Norte $8^{\circ} 48^{\prime}$ Longitud Oeste $75^{\circ} 52^{\prime}$ con respecto al meridiano de Greenwich, con una humedad relativa del $82 \%$, temperatura promedio $28{ }^{\circ} \mathrm{C}$, 
precipitación promedio de $1200 \mathrm{~mm}$ anual y una altura de 12 m.s.n.m. En la investigación se empleo un diseño de bloques completos al azar con cuatro tratamientos y tres repeticiones, los tratamientos fueron:

T0: Cultivo sin competencia.

T1: Cultivo en competencia con una densidad de malezas de 2 plantas $\mathrm{m}^{-2}$.

T3: Cultivo en competencia con una densidad de malezas de 4 plantas $\mathrm{m}^{-2}$.

T4: Cultivo en competencia con una densidad de malezas de 8 plantas $\mathrm{m}^{-2}$.

El área donde se realizo la investigación estuvo comprendida por 3 bloques cada una con 12,8 $\mathrm{m}$ de largo por $5 \mathrm{~m}$ de ancho, dejando $1.0 \mathrm{~m}$ entre bloques y $1.0 \mathrm{~m}$ de márgenes de las mismas, para un área total de $281.2 \mathrm{~m}^{2}$. Para el maíz la distancia de siembra utilizada, fue de $0.20 \mathrm{~m}$ entre planta y planta y de $0.80 \mathrm{~m}$ entre surcos. Para controlar otras malezas durante el ciclo del cultivo, se realizaron limpiezas manuales con machete, en la época de sequía se hicieron riegos frecuentes dejando un día por medio cuando el cultivo lo ameritaba.

La toma de datos se inicio a partir del décimo día de germinado el maíz, cada ocho días hasta la cosecha. Los datos obtenidos fueron tabulados y procesados a través del programa estadístico SAS y un ANACRES donde se hicieron comparaciones del crecimiento del maíz respecto a la caminadora, posteriormente se aplico la prueba de medias DMS y Tukey para determinar diferencias estadísticas entre tratamientos. Las variables evaluadas fueron:

Altura de planta: Con la utilización del flexometro se midió la altura de la planta cada 8 días sobre 3 plantas marcadas.

Área Foliar: Se midió el ancho de la hoja por el largo y se multiplico por la constante 0.75 , este dato se registró cada 8 días.

Número de hojas: Se contó el número hojas que tuvo la planta cada vez que se tomo muestras esto es cada 8 días.
Número y longitud de los entrenudos: Cada 8 días se registró el número de nudos y se midió la distancia desde el primer nudo con lámina foliar y el subsiguiente promediando el valor entre el total de segmentos medidos. Masa seca: Se tomaron cuantas plantas al azar, las que se llevaron al horno de secado durante 72 horas a una temperatura de $70^{\circ} \mathrm{C}$, hasta obtener un peso constante.

Rendimiento: Se cuantificó después de cosechado el cultivo en sus surcos centrales, se pesó y se extrapoló a $\mathrm{Kg} \mathrm{ha}{ }^{-1}$.

Mediante la toma de parámetros e índices fisiotécnicos se realizó los respectivos ANACRES del maíz con respecto a la caminadora y ANACRES a la caminadora. De acuerdo a la metodología propuesta por Radford (1967) citado por Montoya (1990).

\section{RESULTADOS Y DISCUSIÓN}

Se pudo determinar y cuantificar el tiempo de floración masculina, floración femenina y maduración fisiológica del maíz ICA-V-109 las cuales se presentaron a los 41, 43 y 100 días respectivamente. En cuanto a la caminadora (Rottboellia Cochinchinensis L SW. Clayton), se pudo constatar que la germinación de esta fue a los 3 días, el macollamiento se presentó a los 26 días, y la madurez a los 97 días, el número de espigas es de 3 se encontró que las espigas con mayor numero de espiguillas fueron las del tercio superior de la planta, de las cuales solo el 52\% contenían semillas y el $48 \%$ restante estaban vanas, las semillas presentaron un $90 \%$ de germinación, comportamiento que resultó similar a los dos tercios restantes.

\section{Altura de Planta}

Para el cultivo de maíz la mayor altura de planta la presentaron los tratamientos entre los 69 - 84 días después de sembrados (Tabla 1), verificándose que dichos valores fluctúan desde 
Tabla 1. Resultados por tratamientos de valores medios en parámetros de crecimiento en Maíz en competencia con caminadora. (Titular mejor como: Efecto de Diferentes densidades de población de caminadora (R.c.) sobre diferentes variables morfológicas del cultivo del maíz

\begin{tabular}{|c|c|c|c|c|c|c|c|}
\hline Tratamientos & DDE & $\begin{array}{l}\text { Altura de } \\
\text { planta }(\mathrm{cm})\end{array}$ & $\begin{array}{c}\text { Masa } \\
\text { seca }(\mathrm{g})\end{array}$ & $\begin{array}{l}\text { Número } \\
\text { de hojas }\end{array}$ & $\begin{array}{c}\text { Área } \\
\text { foliar } \mathbf{c m}^{2} \\
\end{array}$ & $\begin{array}{c}\text { Número } \\
\text { de nudos }\end{array}$ & $\begin{array}{l}\text { Longitud de } \\
\text { nudos }(\mathrm{cm})\end{array}$ \\
\hline \multirow[t]{7}{*}{ TO } & 16 & $32.5 a$ & & $7.0 \mathrm{a}$ & 694.6a* & $2.8 \mathrm{a}$ & $3.9 a^{*}$ \\
\hline & 24 & $70.3 a$ & & $7.6 a$ & $1907 a$ & $4.4 \mathrm{a}$ & $10.6 a^{*}$ \\
\hline & 39 & $99.1 \mathrm{a}$ & $26.7 a^{*}$ & $8.8 \mathrm{a}$ & $4873 a$ & $6.8 \mathrm{a}$ & $17.0 a^{*}$ \\
\hline & 54 & $134.9 \mathrm{a}$ & $34 . a^{* *}$ & $9.9 \mathrm{a}$ & $7684 a$ & $10.0 \mathrm{a}$ & $19.5 a^{*}$ \\
\hline & 69 & $218.5 a$ & $167.1 \mathrm{a}$ & $11.0 \mathrm{a}$ & $9711 a^{*}$ & $12.8 \mathrm{a}$ & $21.5 a^{*}$ \\
\hline & 84 & $274.5 \mathrm{a}$ & $217.9 a^{*}$ & $13.8 \mathrm{a}$ & 13090a & $11.8 \mathrm{a}$ & $23.2 a^{*}$ \\
\hline & 99 & $283.1 \mathrm{a}$ & $289.1 \mathrm{a}$ & $14.6 \mathrm{a}$ & $14161 \mathrm{a}$ & $12.9 \mathrm{a}$ & $24.3 a^{*}$ \\
\hline \multirow[t]{7}{*}{$\mathrm{T} 1$} & 16 & $33.8 \mathrm{a}$ & & $7.0 \mathrm{a}$ & $644.5 \mathrm{a}$ & $2.8 \mathrm{a}$ & $4.1 \mathrm{a}$ \\
\hline & 24 & $70.2 \mathrm{a}$ & & $7.5 \mathrm{a}$ & $1429 a$ & $4.6 a$ & $6.9 a$ \\
\hline & 39 & $97.1 \mathrm{a}$ & $26.7 \mathrm{a}$ & $8.9 \mathrm{a}$ & $4638 a$ & $5.1 \mathrm{a}$ & $12.2 \mathrm{a}$ \\
\hline & 54 & $123.5 a$ & $34 a$ & $10.1 \mathrm{a}$ & $7796 a$ & $10.1 \mathrm{a}$ & $18.7 \mathrm{a}$ \\
\hline & 69 & $219.9 a$ & $147.5 a$ & $10.7 a$ & 9108ab & $11.7 \mathrm{a}$ & $20.9 a$ \\
\hline & 84 & $267.7 a$ & $170.1 \mathrm{a}$ & 13.3a & $11456 a$ & $10.9 a$ & $21.7 \mathrm{a}$ \\
\hline & 99 & $278.4 \mathrm{a}$ & $261.3 a$ & $14.7 \mathrm{a}$ & 13200a & $12.5 a$ & $22.4 a$ \\
\hline \multirow[t]{7}{*}{$\mathrm{T} 2$} & 16 & $33.1 \mathrm{a}$ & & $6.1 \mathrm{a}$ & $475.5 a$ & $2.9 a$ & $4.4 a$ \\
\hline & 24 & $74.8 \mathrm{a}$ & & $6.8 \mathrm{a}$ & $1651 a$ & $4.5 a$ & $8.1 \mathrm{a}$ \\
\hline & 39 & $95.4 a$ & $23.7 \mathrm{a}$ & $8.5 \mathrm{a}$ & $3529 a$ & $7.3 \mathrm{a}$ & $14.2 \mathrm{a}$ \\
\hline & 54 & $121.9 \mathrm{a}$ & $32.7 \mathrm{a}$ & $9.9 \mathrm{a}$ & $6649 a$ & $9.7 a$ & $18.0 \mathrm{a}$ \\
\hline & 69 & $222.0 \mathrm{a}$ & $130.5 a$ & $10.8 \mathrm{a}$ & $7965 b$ & $10.9 a$ & $20.2 a$ \\
\hline & 84 & $276.7 a$ & 166.6a & $13.5 \mathrm{a}$ & $11338 a$ & $11.0 a$ & $21.3 a$ \\
\hline & 99 & $283.7 \mathrm{a}$ & $252.6 a$ & $14.5 \mathrm{a}$ & $12846 a$ & $13.1 \mathrm{a}$ & $22.4 a$ \\
\hline \multirow[t]{7}{*}{ T3 } & 16 & $27.8 a$ & & $6.4 a$ & $407.2 a$ & $2.9 a^{*}$ & $4.2 \mathrm{a}$ \\
\hline & 24 & $72.9 a$ & & $7.0 \mathrm{a}$ & $1715 a$ & $4.8 \mathrm{a}$ & $10.0 \mathrm{a}$ \\
\hline & 39 & $72.9 a$ & $13.3 \mathrm{a}$ & $9.4 a$ & $3861 a$ & $5.3 a$ & $15.7 \mathrm{a}$ \\
\hline & 54 & $119.9 a$ & $27.9 a$ & $9.9 a$ & $7644 a$ & $10.7 a^{* * *}$ & $17.7 \mathrm{a}$ \\
\hline & 69 & $221.1 \mathrm{a}$ & $117.1 \mathrm{a}$ & $11.3 \mathrm{a}$ & 9039ab & $12.9 a^{*}$ & $19.9 a$ \\
\hline & 84 & $269.5 a$ & 210.6 & $13.2 \mathrm{a}$ & 11680a & $11.5 a^{* * *}$ & $21.1 \mathrm{a}$ \\
\hline & 99 & $281.7 \mathrm{a}$ & $260.1 \mathrm{a}$ & $14.0 \mathrm{a}$ & $12706 a$ & $12.1 \mathrm{a}^{*}$ & $22.5 a$ \\
\hline
\end{tabular}

DDE = Días Después de la Emergencia; * Números con la misma letra no son diferentes de acuerdo con Tukey (0.05)

el T3 al tratamiento testigo (T0), este comportamiento coincide con la floración y Ilenado de grano. Al realizar el análisis estadístico, no se presentaron diferencias significativas entre los tratamientos en ninguna de las épocas de evaluación, sin embargo, el tratamiento T2 expresa los máximos valores desde los 69 - 84 días. Igualmente para la maleza no se presentaron diferencias significativas entre tratamientos (Tabla 2), lo que sugiere que sobre el comportamiento de esta variable no hay ningún efecto de la densidad de siembra de la maleza sobre el cultivo, es decir, que por este parámetro la maleza no se puede considerar competitiva, ya que en ningún estado de desarrollo a lo largo del ciclo del cultivo, la maleza estuvo por encima de las plantas de maíz. 
Tabla 2. Resultados por tratamientos de valores medios en parámetros de crecimiento de Caminadora a diferentes densidades de población. (Considerar revisar este título)

\begin{tabular}{|c|c|c|c|c|c|c|c|}
\hline Tratamientos & DDE & $\begin{array}{c}\text { Altura de } \\
\text { planta }(\mathrm{cm})\end{array}$ & $\begin{array}{c}\text { Masa } \\
\text { seca }(\mathrm{g})\end{array}$ & $\begin{array}{l}\text { Número } \\
\text { de hojas }\end{array}$ & $\begin{array}{c}\text { Área } \\
\text { foliar } \mathbf{c m}^{2} \\
\end{array}$ & $\begin{array}{l}\text { Número } \\
\text { de nudos }\end{array}$ & $\begin{array}{l}\text { Longitud de } \\
\text { nudos }(\mathrm{cm})\end{array}$ \\
\hline \multirow[t]{7}{*}{$\mathrm{T} 1$} & 16 & $33.8 \mathrm{a}$ & & $7.10 \mathrm{a}$ & $91.4 a^{*}$ & $2.9 a$ & $4.10 \mathrm{a}$ \\
\hline & 24 & $70.2 \mathrm{a}$ & $10.1 \mathrm{a}$ & $7.53 a$ & $189.7 a$ & $4.6 a$ & $6.90 a$ \\
\hline & 39 & $97.1 \mathrm{a}$ & $21.2 \mathrm{a}$ & $8.90 a$ & 516.3a & $5.1 \mathrm{a}$ & $16.20 \mathrm{a}$ \\
\hline & 54 & $123.5 a$ & $30.2 \mathrm{a}$ & $10.10 \mathrm{a}$ & $775.7 a$ & $10.1 \mathrm{a}$ & $18.70 \mathrm{a}$ \\
\hline & 69 & $219.9 a$ & $147.5 \mathrm{a}$ & 10.70a & $848.3 a^{*}$ & $11.7 \mathrm{a}$ & $20.93 a$ \\
\hline & 84 & 267.7 & $170.1 \mathrm{a}$ & 13.30a & $863.7 \mathrm{a}$ & $10.9 a$ & $21.67 a$ \\
\hline & 99 & $278.4 a$ & $261.3 \mathrm{a}$ & $14.30 \mathrm{a}$ & $925.6 a$ & $11.5 \mathrm{a}$ & $22.70 \mathrm{a}$ \\
\hline \multirow[t]{7}{*}{$\mathrm{T} 2$} & 16 & $33.1 \mathrm{a}$ & & $6.10 a$ & $74.5 a$ & $2.9 a$ & $4.40 \mathrm{a}$ \\
\hline & 24 & $74.8 \mathrm{a}$ & $13.1 \mathrm{a}$ & $6.80 a$ & $240.7 a$ & $4.5 a$ & $8.10 \mathrm{a}$ \\
\hline & 39 & $95.4 a$ & $23.7 a^{*}$ & $8.50 a$ & $421.4 a$ & 7.3a & $14.20 \mathrm{a}$ \\
\hline & 54 & $121.9 a$ & $32.7 a^{* * *}$ & $9.90 a$ & $674.5 a$ & $9.7 \mathrm{a}$ & $18.00 \mathrm{a}$ \\
\hline & 69 & $222.0 a$ & $130.5 a$ & 10.80a & $739.1 \mathrm{a}$ & $10.9 a$ & $20.20 a$ \\
\hline & 84 & $276.7 a$ & 166.6a* & 13.50a & $841.1 \mathrm{a}$ & 11.0a & $21.30 \mathrm{a}$ \\
\hline & 99 & $287.7 \mathrm{a}$ & $252.6 \mathrm{a}$ & $14.50 \mathrm{a}$ & 883.3a & $13.1 \mathrm{a}$ & $22.40 \mathrm{a}$ \\
\hline \multirow[t]{7}{*}{$\mathrm{T} 3$} & 16 & $27.8 \mathrm{a}$ & & $6.40 a$ & $64.2 \mathrm{a}$ & $2.9 a^{*}$ & $4.20 \mathrm{a}$ \\
\hline & 24 & $72.9 a$ & $10.0 \mathrm{a}$ & $7.00 \mathrm{a}$ & $246.2 a$ & $4.8 \mathrm{a}$ & $10.00 \mathrm{a}$ \\
\hline & 39 & $72.9 a$ & $13.3 \mathrm{a}$ & $9.40 \mathrm{a}$ & $410.5 a$ & $5.3 a$ & $15.70 \mathrm{a}$ \\
\hline & 54 & 119.9a & $27.9 a$ & $9.90 a$ & $770.1 \mathrm{a}$ & $10.7 a^{* * *}$ & $17.70 \mathrm{a}$ \\
\hline & 69 & $221.1 \mathrm{a}$ & $117.2 \mathrm{a}$ & 11.30a & 797.3a & $12.9 a^{*}$ & $19.90 a$ \\
\hline & 84 & $269.5 a$ & $21.6 a$ & 13.20a & $885.1 \mathrm{a}$ & $11.5 \mathrm{a}^{* * *}$ & $21.10 \mathrm{a}$ \\
\hline & 99 & $281.7 \mathrm{a}$ & $360.8 \mathrm{a}$ & 14.10a & $903.5 a$ & $12.1 a^{*}$ & $22.50 \mathrm{a}$ \\
\hline
\end{tabular}

DDE = Días Después de la Emergencia; ${ }^{*}$ Números con la misma letra no son diferentes de acuerdo con Tukey (0.05)

\section{Número de hojas}

No se presentaron diferencias significativas (Tabla 1), entre la densidad de siembra tanto para el cultivo de maíz y la planta de caminadora en ninguna de las épocas de evaluación. La mayor tasa de emisión de hoja ocurrió entre los 54 - 90 días después de sembrada, lo cual demuestra que es una maleza con una alta capacidad para la emisión de área foliar (Tabla 2), característica muy importante para la captación de luz solar, lo cual indica que es una planta que por la emisión de hojas, tiene una constante traslocación de foto-asimilados. La producción de gran cantidad de hojas en estas épocas es un indicativo de una alta tasa de crecimiento relativo, lo que hace que el período vegetativo sea corto con relación al productivo y por lo tanto se esperaría una mayor producción de semillas.

\section{Área foliar}

En el cultivo de maíz se observó diferencia significativa a los 16 y 69 días después de siembra (Tabla 1) en la formación de área foliar entre tratamientos siendo el tratamiento control el que presentó los valores máximos. A los 16 y 69 días después de siembra se presentó diferencia significativa en la maleza entre los tratamientos siendo el tratamiento T1el que expresó los máximos valores, cabe resaltar el comportamiento de T3 que expreso los máximos valores a los 24, 54 y 84 días después de la siembra (Tabla 2), lo cual indica 
que en este estado es donde la maleza presenta su mayor capacidad fotosintética y mayor capacidad de almacenamiento de fotoasimilados para luego enviar a los vertederos, esto demuestra que hay efecto de las diferentes densidades de población de la caminadora sobre el área foliar de la planta de maíz, al observar los resultados alcanzados en área foliar por la mezcla $\left(925,57 \mathrm{~cm}^{2}\right)$, y comparar éstos con malezas altamente competitivas como el arroz rojo $\left(319,88 \mathrm{~cm}^{2}\right)$, indica que la caminadora es una maleza altamente competitiva y eficiente desde el punto de vista fotosintético, lo que la convierte en una planta adaptada y con características competitivas frente al cultivo de maíz (Montealegre, 2000).

\section{Número de nudos}

El Anava en maíz arrojo diferencias significativas a los 16, 69 y 99 dias y altamente significativa a los 54 y 84 días entre tratamientos (Tabla 1). Donde el tratamiento T3 expresa sus máximos valores desde los 54 a los 69 días. En cuanto a la caminadora se presentan diferencias significativas a los 16 y 99 días y altamente significativa desde los 54 a los 84 días, y es precisamente en esta época en donde el tratamiento T3 el que formó mayor número de nudos por planta (Tabla 2), observándose que la maleza sembrada a 8 malezas $\mathrm{m}^{-2}$ se muestra mas competitiva debido a que dispone de menor espacio y en donde debe exigirse y llegar más rápido a la producción de estructuras reproductivas y posteriormente su senescencia.

\section{Longitud de nudos}

En maíz se presentaron diferencias significativas en todas las épocas de evaluación a excepción del día 69 en donde el tratamiento testigo muestra los valores más altos que los demás tratamientos; (Tabla 1), con igual comportamiento del maíz, la maleza presento diferencia significativa entre tratamientos, presentando el tratamiento T1 los valores máximos a través de todas las evaluaciones. Esto demuestra que la maleza es altamente competitiva en cuanto a esta variable viéndose reflejada en una alta tasa de crecimiento (Tabla 2).

\section{Masa seca}

Los resultados en maíz demuestran que hay diferencias significativas a los 39 y 84 días y altamente significativa a los 54 días en donde el tratamiento testigo registró los valores más altos (Tabla 1), presentándose una tendencia creciente en todos los tratamientos, mostrando la habilidad de las plantas sin competencia en el proceso de acumulación de foto-asimilados, la tendencia creciente a partir de los 54 días después de siembra se debe a la distribución de foto-asimilados hacia los órganos de demanda como flores y frutos puesto que a partir de este punto se da inicio a la fase reproductiva de las plantas. En cuanto a los resultados de la caminadora se observó que hay diferencias significativas a los 39 y 84 días y altamente significativa a los 54 días en donde el T2 presentó los valores más altos y en cuanto a los 84 días el T3 fue el que expresó la mayor acumulación de materia seca (Tabla 2), esto quizás se debió a la densidad de población a menos distancia entre plantas, mayor cubrimiento por parte de la caminadora. Este comportamiento coincide con lo expuesto por Jarma (1999), en relación al área foliar y masa seca en habichuela, siendo estas directamente proporcionales en las épocas de evaluación donde se presentaron los grados de significancia, lo que también se ve expresado en los índices fisicotécnicos como TAN, TAC y RAF.

\section{Tasa Absoluta de Crecimiento (TAC)}

En maíz los resultados muestran que se presentó diferencia significativa a los 84 días entre tratamientos, la TAC presentó menos variabilidad para el tratamiento testigo y obtuvo su mayor variación para el tratamiento T3 (8 plantas $\mathrm{m}^{-2}$ ) mostrándose con ello la habilidad de las plantas bajo el tratamiento testigo (sin malezas) para acumular biomasa en función del tiempo (Tabla 3). Mientras que en el T3 la maleza se mostró más competitiva 
Tabla 3. Resultados de valores medios en análisis de crecimiento de Maíz en competencia con caminadora (Revisar Título).

\begin{tabular}{|c|c|c|c|c|c|c|c|c|}
\hline Tratamientos & DDE & TAC & TRC & TAN & TCC & DAF & RAF & IAF \\
\hline \multirow[t]{7}{*}{$\mathrm{TO}$} & 16 & $2.67 a$ & $0.8 \mathrm{a}$ & $0.00001 \mathrm{a}$ & $33.3 a$ & $3.0 \mathrm{a}$ & $188.4 a$ & $0.43 a$ \\
\hline & 24 & $3.00 \mathrm{a}$ & $0.9 a$ & 0.0008a & $39.6 a$ & $3.0 \mathrm{a}$ & $200.2 a$ & $1.19 a$ \\
\hline & 39 & $4.60 \mathrm{a}$ & $0.1 \mathrm{a}$ & $0.0087 a$ & $47.4 \mathrm{a}$ & $13.9 a$ & $301.9 a$ & $3.00 \mathrm{a}$ \\
\hline & 54 & $7.60 a$ & $0.1 \mathrm{a}$ & $0.0096 a$ & $49.7 a$ & $13.2 \mathrm{a}$ & $460.2 \mathrm{a}$ & $4.80 \mathrm{a}$ \\
\hline & 69 & $9.90 a$ & $0.1 \mathrm{a}$ & 0.001 & $55.5 \mathrm{a}$ & $9.5 a$ & $68.3 a$ & $6.1 a^{*}$ \\
\hline & 84 & $3.4 a^{*}$ & $0.01 \mathrm{a}$ & $0.0002 \mathrm{a}$ & $21.1 \mathrm{a}$ & $8.2 \mathrm{a}$ & $74.3 a$ & $8.20 \mathrm{a}$ \\
\hline & 99 & $4.70 \mathrm{a}$ & $0.2 \mathrm{a}$ & $0.0003 a$ & $29.7 a$ & $8.8 \mathrm{a}$ & $56.9 a$ & $8.80 a$ \\
\hline \multirow[t]{7}{*}{$\mathrm{T} 1$} & 16 & $1.30 \mathrm{a}$ & $0.6 \mathrm{a}$ & $0.000009 a$ & $26.5 a$ & $1.0 \mathrm{a}$ & $163.1 \mathrm{a}$ & $0.40 \mathrm{a}$ \\
\hline & 24 & $2.20 \mathrm{a}$ & $0.8 \mathrm{a}$ & $0.00008 a$ & $33.9 a$ & $2.0 \mathrm{a}$ & $173.4 \mathrm{a}$ & $0.90 \mathrm{a}$ \\
\hline & 39 & $3.00 \mathrm{a}$ & $0.9 a$ & $0.00074 a^{*}$ & $37.5 \mathrm{a}$ & $15.0 \mathrm{a}$ & 277.5 & $2.90 \mathrm{a}$ \\
\hline & 54 & $6.40 a$ & $0.1 \mathrm{a}$ & $0.00085 a^{*}$ & $40.8 a$ & $14.8 \mathrm{a}$ & $375.6 \mathrm{a}$ & $4.90 \mathrm{a}$ \\
\hline & 69 & $7.80 \mathrm{a}$ & $0.1 \mathrm{a}$ & 0.0009a* & $48.9 a$ & $6.1 \mathrm{a}$ & $63.7 a$ & $5.70 \mathrm{a}$ \\
\hline & 84 & $1.50 \mathrm{a}$ & $0.006 a$ & $0.0001 \mathrm{a}$ & $9.4 a$ & 11.0a & $75.6 \mathrm{a}$ & $7.20 \mathrm{a}$ \\
\hline & 99 & $6.10 \mathrm{a}$ & $0.02 \mathrm{a}$ & $0.0004 a$ & 38.0a & $8.2 \mathrm{a}$ & $79.7 a$ & $8.20 \mathrm{a}$ \\
\hline \multirow[t]{7}{*}{$\mathrm{T} 2$} & 16 & $1.30 \mathrm{a}$ & $0.4 a$ & $0.000006 a$ & $26.1 \mathrm{a}$ & $1.0 \mathrm{a}$ & $140.3 a$ & $0.30 \mathrm{a}$ \\
\hline & 24 & $2.00 \mathrm{a}$ & $0.8 a$ & $0.00007 a$ & $30.0 a$ & $2.9 \mathrm{a}$ & $152.6 a$ & $1.00 \mathrm{a}$ \\
\hline & 39 & $2.30 \mathrm{a}$ & $0.9 a$ & $0.00065 a$ & $30.2 \mathrm{a}$ & $9.1 \mathrm{a}$ & 213.0a* & $2.20 \mathrm{a}$ \\
\hline & 54 & $6.00 \mathrm{a}$ & $0.9 a$ & $0.00063 a$ & $40.0 a$ & $14.3 \mathrm{a}$ & $295.3 a^{* * *}$ & $4.20 \mathrm{a}$ \\
\hline & 69 & $6.50 a$ & $0.1 \mathrm{a}^{* * *}$ & 0.0009a & $40.1 \mathrm{a}$ & $6.2 \mathrm{a}$ & $61.5 a$ & $5.00 a$ \\
\hline & 84 & $2.40 a$ & $0.016 a$ & $0.0002 a$ & $15.0 \mathrm{a}$ & $15.8 \mathrm{a}$ & $69 a^{*}$ & $7.10 a$ \\
\hline & 99 & $5.70 \mathrm{a}$ & $0.02 \mathrm{a}^{* * *}$ & $0.0004 a$ & $35.8 \mathrm{a}$ & $7.1 \mathrm{a}$ & $52.8 a^{*}$ & $8.00 \mathrm{a}$ \\
\hline \multirow[t]{7}{*}{ T3 } & 16 & $1.20 \mathrm{a}$ & $0.2 \mathrm{a}$ & $0.000004 a$ & $23.9 a$ & $3.0 \mathrm{a}$ & $160.1 \mathrm{a}$ & $0.20 \mathrm{a}$ \\
\hline & 24 & $1.40 \mathrm{a}$ & $0.7 a$ & $0.00004 a$ & $26.9 a$ & $3.3 \mathrm{a}$ & $169.1 \mathrm{a}$ & $1.10 \mathrm{a}$ \\
\hline & 39 & $2.00 \mathrm{a}$ & $0.8 a$ & $0.00049 a$ & $29.2 \mathrm{a}$ & $10.1 \mathrm{a}$ & $400.6 a$ & $2.40 \mathrm{a}$ \\
\hline & 54 & $5.40 \mathrm{a}$ & $0.9 a$ & $0.00052 \mathrm{a}$ & $31.2 \mathrm{a}$ & $17.7 \mathrm{a}$ & $360.9 a$ & $4.80 \mathrm{a}$ \\
\hline & 69 & $5.90 a$ & $0.1 \mathrm{a}^{* * *}$ & $0.0007 a$ & $37.2 \mathrm{a}$ & $6.5 a$ & $81.2 \mathrm{a}$ & $5.60 a$ \\
\hline & 84 & $6.20 \mathrm{a}$ & $0.034 a$ & 0.0006a & $38.9 a$ & $12.4 \mathrm{a}$ & $67.5 a$ & $7.30 \mathrm{a}$ \\
\hline & 99 & 10.00a & $0.4^{* * *}$ & $0.0007 a$ & $62.6 a$ & $4.8 \mathrm{a}$ & $39.4 a$ & $7.90 \mathrm{a}$ \\
\hline
\end{tabular}

DDE = Días Después de la Emergencia; * Números con la misma letra no son diferentes de acuerdo con Tukey (0.05)

con relación al cultivo, expresando su TAC más alta entre los 56 días después de la siembra hasta y 84 días después de la siembra cuando el cultivo está en plena fase de floración y llenado del grano, todo ocasionado por estar a menor distancia. En cuanto a la caminadora, los resultados indican que se presentó diferencia significativa a los 54 y 84 días después de siembra (Tabla 4), se puede apreciar que el tratamiento 3 (8 plantas $\mathrm{m}^{-2}$ ) presenta un comportamiento similar al T3 de la TAC del cultivo, demostrando la habilidad de la maleza a esta densidad, de competir con el cultivo para acumular biomasa en función constante durante de tiempo. Además existe una relación directa con el área foliar y la masa seca en ese mismo estado de desarrollo en el cual la maleza presenta mayor grado de área foliar a lo largo de todo su ciclo vegetativo, lo cual coincide con un estudio realizado en 
Argentina por Juan et al. (2003) al evaluar competencia entre Euphorbia dentata y Soya, donde las plantas de cultivo sufren el efecto de las mayores densidades de la maleza en cuanto a la acumulación de materia seca, de igual forma coincidió con lo encontrado por Rivera (2006), al evaluar competencia entre Stemodia durantifolia (L) con maíz en el Medio Sinú.

\section{Tasa Relativa de Crecimiento (TRC)}

Los resultados observados en maíz muestran diferencias altamente significativas entre tratamientos a los 69 y 99 días (Tabla 3). Además, el comportamiento de la TRC es similar en los tratamientos T3 y T2, siendo que todos los tratamientos siguen una tendencia acorde con lo observado por Wallace y
Munger (No aparece en Bibliografía) (1995), quienes afirman que la TRC presenta otros valores que van disminuyendo con la edad de la planta. Aquí los tratamientos T3 y T2 presentan valores altos, luego inician una tendencia constante y luego decrecen mostrando el efecto de la distancia entre plantas del cultivo sobre el crecimiento de la maleza. En cuanto a la caminadora, los resultados muestran que se presentó diferencia altamente significativa entre los tratamientos a los 69 días y a los 99, aunque el tratamiento T3 fue quien presentó los valores de TRC más altos a los 56 días después de siembra, demostrando con ello la habilidad de la maleza para crecer cuando se manejan estas densidades (Tabla 4).

Tabla 4. Resultados de valores medios en análisis de crecimiento en Caminadora a diferentes densidades de población compitiendo con maíz. (Revisar título)

\begin{tabular}{|c|c|c|c|c|c|c|c|c|}
\hline Tratamientos & DDE & TAC & TRC & TAN & TCC & DAF & RAF & IAF \\
\hline \multirow[t]{7}{*}{$\mathrm{T} 1$} & 16 & $4.0 \mathrm{a}$ & $0.6 a$ & $0.0027 a$ & $9.0 \mathrm{a}$ & $0.97 a$ & $17.70 \mathrm{a}$ & $0.02 b$ \\
\hline & 24 & $4.6 \mathrm{a}$ & $0.8 \mathrm{a}$ & $0.0038 a$ & $9.6 \mathrm{a}$ & $0.10 \mathrm{c}$ & 18.60a & $0.05 c$ \\
\hline & 39 & $5.5 \mathrm{a}$ & $0.9 a$ & $0.0046 a$ & $10.0 \mathrm{a}$ & $0.60 a$ & $32.60 \mathrm{a}$ & $0.13 b$ \\
\hline & 54 & $6.4 \mathrm{a}$ & $0.96 a$ & $0.0087 a^{*}$ & $13.4 \mathrm{a}$ & $0.50 \mathrm{c}$ & $37.70 a^{*}$ & $0.19 c$ \\
\hline & 69 & $7.8 \mathrm{a}$ & $0.11 \mathrm{a}$ & $0.009 a$ & $19.5 a$ & $0.10 a$ & $5.90 a$ & $0.21 \mathrm{c}$ \\
\hline & 84 & $1.5 \mathrm{a}$ & $0.006 a$ & $0.001 \mathrm{a}$ & $3.8 \mathrm{a}$ & $0.03 b$ & $5.70 a^{*}$ & $0.22 \mathrm{c}$ \\
\hline & 99 & $6.1 \mathrm{a}$ & $0.02 a$ & $0.006 a$ & $15.2 \mathrm{a}$ & $0.10 a$ & $5.60 \mathrm{a}$ & $0.23 c$ \\
\hline \multirow[t]{7}{*}{$\mathrm{T} 2$} & 16 & $3.6 a$ & $0.5 \mathrm{a}$ & $0.0038 a$ & $14.2 \mathrm{a}$ & $0.30 a$ & $14.90 a$ & $0.04 a b$ \\
\hline & 24 & $5.6 a$ & $0.6 a$ & $0.0043 a$ & $16.4 a$ & $0.33 b$ & $15.30 \mathrm{a}$ & $0.12 b$ \\
\hline & 39 & $3.7 \mathrm{a}$ & $0.7 \mathrm{a}$ & $0.0052 \mathrm{a}$ & $19.2 \mathrm{a}$ & $0.70 a$ & $24.50 a$ & $0.21 b$ \\
\hline & 54 & $4.3 \mathrm{a}$ & $0.88 a$ & $0.0083 a$ & $27.6 a$ & $0.95 b$ & $30.40 a$ & $0.34 b$ \\
\hline & 69 & $6.5 a$ & $0.10 a$ & $0.009 a$ & $32.6 a$ & $0.20 a$ & $5.70 \mathrm{a}$ & $0.37 b$ \\
\hline & 84 & $2.4 \mathrm{a}$ & $0.015 a$ & $0.003 a^{*}$ & $12.0 \mathrm{a}$ & $0.40 \mathrm{ab}$ & $5.11 \mathrm{a}$ & $0.42 b$ \\
\hline & 99 & $5.7 \mathrm{a}$ & $0.03 a$ & $0.006 a$ & $28.7 \mathrm{a}$ & $0.16 a$ & $3.60 a$ & $0.44 b$ \\
\hline \multirow[t]{7}{*}{ T3 } & 16 & $4.1 \mathrm{a}$ & $0.7 a$ & $0.0048 a$ & $18.1 \mathrm{a}$ & $0.63 a$ & $11.70 a$ & $0.06 a$ \\
\hline & 24 & $5.6 a$ & $0.8 \mathrm{a}$ & $0.0057 a$ & $20.4 a$ & $0.73 a^{*}$ & 11.90a & $0.25 a^{*}$ \\
\hline & 39 & $7.4 \mathrm{a}$ & $0.8 \mathrm{a}$ & $0.0060 a$ & $28.3 a$ & $0.63 a$ & $42.20 \mathrm{a}$ & $0.41 a^{*}$ \\
\hline & 54 & $9.6 a^{*}$ & $0.1 \mathrm{a}$ & $0.0063 a$ & $48.4 a$ & $2.70 \mathrm{a}^{*}$ & $54.80 \mathrm{a}$ & $0.77 a^{* * *}$ \\
\hline & 69 & $5.9 a$ & $0.11 \mathrm{a}^{*}$ & $0.007 a$ & $59.5 a$ & $0.21 \mathrm{a}$ & $7.30 \mathrm{a}$ & $0.80 a$ \\
\hline & 84 & $6.2 a^{*}$ & $0.034 a$ & $0.007 a$ & $62.3 a$ & $0.65 a^{*}$ & $5.10 \mathrm{a}$ & $0.89 a$ \\
\hline & 99 & $10.0 \mathrm{a}$ & $0.038 a^{*}$ & $0.0011 a^{*}$ & $100.2 \mathrm{a}$ & $0.14 a$ & $2.80 \mathrm{a}$ & $0.90 a^{* * *}$ \\
\hline
\end{tabular}

DDE = Días Después de la Emergencia; * Números con la misma letra no son diferentes de acuerdo con Tukey (0.05) 


\section{Tasa de Asimilación Neta (TAN)}

La investigación realizada muestra el comportamiento de la tasa de asimilación neta, se observa que el tratamiento testigo en el cultivo mostró mayor eficiencia foto-sintética hacia los 56 días después de siembra, con una tendencia diferente entre los demás tratamientos (Tabla 3). Esta respuesta es muy similar con la obtenida con Gómez et al. (1999) en papa (Solanum tuberosum) quien argumenta que la relación entre la masa seca de la planta y el área foliar es lineal en los estados iniciales de crecimiento más no en los estados finales, donde el desarrollo del área foliar puede exceder aumentos en masa seca y viceversa. Se muestra que el período entre los 40 a los 70 días hubo diferencia significativa con un promedio de $0.00260 \mathrm{~g} \mathrm{~cm}^{-2}$ día $^{-1}$ para el tratamiento T1 y 0.00036 y $0.00090 \mathrm{~g} \mathrm{~cm}^{-2} \mathrm{día}^{-1}$ en los tratamientos T2 y T3 respectivamente. En Caminadora, los resultados demuestran que se presentó diferencia significativa para los 54, 84 y 99 días de evaluación entre los tratamientos de caminadora. Se evidencia el comportamiento similar de los tratamientos T1 y T2 y con una tendencia constante y creciente al T3, al comparar esta, con la TAC indica que el área foliar aprovecha al máximo la radiación y la utiliza para un alta tasa de asimilación y la habilidad de las plantas para competir en términos de respuesta fisiológicas y morfológicas influenciada por el ambiente como lo encontraron Eagles y Toman (1986) citado por Marin, (1989) en análisis de crecimiento en Canavalia ensiformis.

\section{Tasa de Crecimiento (TCC)}

Se observó un crecimiento ligeramente progresivo a los 56 y 84 días después de siembra, según el análisis no se presentaron diferencia significativa entre tratamientos, siendo el tratamiento testigo quien expresa los máximos valores de TCC seguido por el tratamiento $\mathrm{T} 2$ y se observa el comportamiento constante y progresivo del T3 (Tabla 3). En lo referente a la caminadora los resultados muestran que no hubo diferencia significativa, siendo el tratamiento T3 quien mostrará los mayores valores a los 54-70 y 84 días con relación al resto de los tratamientos (Tabla 4).

\section{Duración de Área Foliar (DAF)}

Se evidencia un comportamiento ligero y progresivo desde los 40 a los 60 días desde los 60 a los 100 días, donde los resultados no indican la presencia de diferencia significativa entre los tratamientos. No obstante, el tratamiento testigo es el que tiene comportamiento constante a través del tiempo. En la maleza los resultados muestran diferencias significativas a los 24, 54 y 84 días, siendo el tratamiento T3 quien expresó los valores más altos de DAF (Tabla 4), demostrando la habilidad de las plantas sometidas a estas densidades de población para mantener más área foliar durante el tiempo lo cual se traduce en una mayor captación de radiación solar.

\section{Relación del Área Foliar (RAF)}

En maíz se presentaron diferencias significativas a los 39, 84 y 99 días de evaluación y diferencias altamente significativas a los 54 días en donde el T3 presentó los valores más altos de RAF (Tabla 3). En cuanto a la caminadora los resultados muestran diferencias altamente significativas a los 54 y 84 días resaltando el alto grado de relación de área foliar del tratamiento T2 cuya relación estuvo muy cercana a la floración y llenado de grano, comportamiento seguido por los tratamientos T1 y T3 (Tabla 4).

\section{Índice de Área Foliar (IAF): Índice de Área Foliar (IAF)}

Mostró un comportamiento constante de la IAF donde los tratamientos expresan sus máximos valores a los 80 días, siendo el tratamiento control el que presenta los valores más altos de IAF a través del tiempo. Esto indica que el maíz presentó mayor exposición de las hojas a la radiación solar, lo cual se traduce en una mayor producción de foto-asimilados para la 
planta, que concuerda con los valores alcanzados por la TAN correspondientes a esta época (Tablas 3 y 4).

\section{Largo de la mazorca}

No presentaron diferencias significativas entre tratamientos en longitud de mazorca durante la evaluación, sin embargo, se observó que las plantas de maíz sometidas a una mayor competencia con las malezas, presentaron los valores más bajos (Tabla 5).

\section{Grosor de mazorca}

Los resultados muestran que no hubo diferencias significativas entre tratamientos, siendo el tratamiento T3 quien presentó el valor más bajo $(39.67 \mathrm{~cm}$ ) en promedio, donde se ve reflejada la eficiencia fotosintética y productiva de la caminadora y su efecto en competencia con el cultivo de maíz.

\section{Rendimiento}

En la tabla 5 se pueden apreciar los rendimientos obtenidos por tratamiento, donde se muestra que hubo diferencias altamente significativas entre el testigo y los tratamientos T1, T2 y T3 equivalentes $514 \mathrm{Kg}$ (13.06\%), $1147 \mathrm{Kg}(28.89 \%)$ y $1460 \mathrm{Kg}(37 \%)$, respectivamente. Estos resultados demuestran que el período de mayor interferencia o agresividad de la maleza se presenta entre los 54-56 días, coincidiendo con la fase de floración e inicio de formación del grano en las plantas de maíz, siendo la etapa donde se causa mayor efecto sobre el cultivo y su proceso de desarrollo.

Tabla 5. Resultados de valores medios en parámetros de rendimiento en maíz en competencia con caminadora. (Revisar Título)

\begin{tabular}{cccc}
\hline Tratamientos & Longitud de mazorca $(\mathbf{c m})$ & Grosor de mazorca $(\mathbf{c m})$ & Rendimiento $\left(\right.$ Kgha $\left.^{-1}\right)$ \\
\hline TO & $16.3 \mathrm{a}$ & $43.5 \mathrm{a}$ & 3980 \\
T1 & $15.9 \mathrm{a}$ & $42.9 \mathrm{a}$ & 3470 \\
T2 & $15.7 \mathrm{a}$ & $42.6 \mathrm{a}$ & 2830 \\
T3 & $14.7 \mathrm{a}$ & $40.0 \mathrm{a}$ & 2520 \\
\hline
\end{tabular}

* Números con la misma letra no son diferentes de acuerdo con Tukey (0.05)

\section{CONCLUSIONES}

- La máxima expresión fenológica de la caminadora (Rottboellia cochinchinensis) teniendo en cuenta la TAN (tasa de asimilación neta), TAC (tasa absoluta de crecimiento), TRC (tasa relativa de crecimiento), RAF (relación de área foliar), DAF (duración de área foliar), IAF (índice de área foliar), fue a los 60 días después de la siembra.

- Los mayores valores de la RAF junto con la TRC reflejan la condición de eficiencia en la captación de luz y productividad de fotoasimilados de la caminadora (Rottboellia cochinchinensis).

- La mayor expresión fenológica del maíz teniendo en cuenta la TAC y la RAF fue a los 60 días después de la siembra.

- La competencia de la caminadora (Rottboellia cochinchinensis) en los tratamientos T1 (2 plantas $\mathrm{m}^{-2}$ ), T2 (4 plantas $\mathrm{m}^{-2}$ ) y T3 (8 plantas $\left.\mathrm{m}^{-2}\right)$, ocasionó reducciones del rendimiento del maíz del $13.06,28.89$ y $37 \%$, en comparación con el tratamiento control (maíz sin competencia). 


\section{AGRADECIMIENTOS}

Los autores, expresan especialmente a los Ingenieros Agrónomos, Edgar Antonio Angulo Gil, y José Gregorio Manjares Núñez, sus agradecimientos al igual que a la Universidad de Córdoba, a la Facultad de Ciencias Agrícolas; por todo el apoyo prestado en la realización del estudio.

\section{BIBLIOGRAFÍA}

Arrieta, J. 1996. La Ciencia de las Arvenses y su Prospectiva. Memorias del Curso Introducción a la Dinámica de Plagas. C.I. Tibaitatá, Corpoica, Mosquera, p 35-37 (páginas consultadas o páginas totales del documento)

Cárdenas, J.; Reyes, C.; Doll, J. y Pardo,F. 1972. Tropical weeds; arvenses tropicales. Vol. 1. ICA. Bogotá. p3-7

CIAT. 1996. Control y Manejo de Malezas. Guía de estudio. CIAT, Palmira, p23-62

Gómez, C.; Buitrago, C.; Cante, M. y Huertas, B. 1999. Ecofisiología de la papa (Solanum tuberosum) utilizada para consumo fresco y para la industria. Revista Comalfi 26:42-55

Jarma, A. 1999. Respuesta del crecimiento de habichuela (Phaseolus vulgaris. L. var. Blue Lake.) a tres niveles de radiación incidente. Revista Comalfi 26:62-73

Juan, V.; Saint, A. y Fernández, R. 2003. Competencia de Lecheron (Euphorbia dentata) en Soya. Revista Sociedade Brasileira da Ciencia das Plantas Danihnas 205:175-180
Klingman, G. y Ashton, F. 1989. Estudio de las plantas nocivas. Principios y Prácticas. Editorial Limusa, México D.F., p25

López y Rivero. 1996. control en cultivos de clima cálido. (Indicar el Tipo de Documento y la editorial) Bogotá, Colombia, p23-62

Montealegre, F. 2000. Competencia intra e interespecífica de malezas con el arroz. Revista Ventana al campo 3(1):44-45

Montoya, R. 1990 Análisis de Crecimiento y Desarrollo de la Pitaya amarilla. Tesis M. Sc., Universidad Nacional de Colombia, Bogotá

Rivera, J. 2006. Caracterización biológica de Stemodia durantifolia (L) Sw. en condiciones del Sinú Medio. Tesis M. Sc., Universidad Nacional de Colombia, Bogotá

Rivero, L. 1996. Aspectos importantes del manejo de malezas en el cultivo de Maíz. Descripción de algunas malezas importantes. (editorial) México D.F. p24-36 